\title{
Waste tire rubber in polymer blends: a review on the evolution, properties and future
}

\begin{abstract}
This review addresses the progress in waste tire recycling with a particular attention to incorporation of waste tire rubber (WTR) into polymeric matrices. Methods of waste tire downsizing, importance of WTR characterization and current practice of WTR modification has been emphasized. Detailed discussion on influence of WTR size, loading, modification, compatibilization and crosslinking on the rheological, mechanical and thermal properties of rubber, thermoplastic and thermoplastic elastomer blends utilizing WTR has been reported. By far, thermoplastic elastomer blends; though still in its infancy; has shown the most promising properties balance which is capable of commercialization. Rubber/WTR blends also show ease of processing and acceptable properties. Thermoplastic/WTR blends suffers in term of toughness and elongation at break. However, the waste thermoplastic/WTR is a viable solution to address polymeric waste problem. Review also highlights the lack of studies concentrating on dynamic mechanical, aging, thermal and swelling properties of WTR polymeric blends.
\end{abstract}

Keyword: Recycling; Waste tire; Ground tire rubber; Reclaimed tire rubber; GTR polymer blends; Compatibilization 